\title{
A brief review of pemphigus vulgaris
}

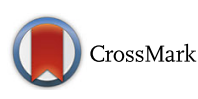

\author{
William J. Sanders ${ }^{1,2}$
}

\begin{abstract}
Pemphigus vulgaris is an autoimmune disorder which presents with painful mucocutaneous blisters and erosions. On the skin, they are flaccid bullae or erosions, and on the mucosa, they present as erosions. This disease is rare but is devastating to those who have it; it also is related-perhaps genetically - to other autoimmune conditions. This is to say that a patient can develop pemphigus vulgaris if they have thyroiditis or diabetes mellitus. Biopsy is needed to obtain histopathological evidence of the breakdown of intercellular connections due to the autoimmune attack on components of desmosomes, which are responsible for intercellular integrity above the basement membrane. When these desmosomes are attacked, loss of connection ensues, and the cells break apart at these connections; this leads to fluid buildup, seen grossly as bullae. Treatment of the disease is difficult and sometimes unsafe. For decades, the mainstay of treatment has been glucocorticoids followed by other drugs. Unfortunately, these drugs are systemically absorbed, and the side effect profile can be unfavorable. In the past several years however, more innovative treatments have emerged that may help ease the cost and safety burden to patients. This review highlights the major points about pemphigus vulgaris, its pathophysiology, and its treatment.
\end{abstract}

Keywords: Pemphigus vulgaris, Autoimmune, Acantholysis, Mucosal, Skin, Erosion, Bullae

\section{Background}

Pemphigus vulgaris (PV) is a debilitating dermatologic condition that is autoimmune in nature. It can be life threatening and can have a mortality rate between 5 and 15\% (Razzaque Ahmed and Moy 1982). Usually, mortality has been associated with skin infections or pneumonias as a result of the structural damage caused by PV. It can present with lesions on the mucosal and skin surfaces (Mustafa et al. 2015). Usually, the oral mucosa will be the first to present with lesions. The reason PV is so devastating is that often times the treatment for it causes further medical problems and conditions. However, as in all of medicine, the treatment protocol is becoming more streamlined and advanced as we learn more about the disease. There are emerging options for treatment that will benefit patients with the disease while decreasing the likelihood of creating or exacerbating a new medical condition concomitant to that of PV.

\footnotetext{
Correspondence: Williamsan@pcom.edu

${ }^{1}$ Georgia Campus-Philadelphia College of Osteopathic Medicine (GA-PCOM), Suwanee, GA 30024, USA

${ }^{2}$ Houston Medical Center, Warner Robins, GA 31088, USA
}

\section{Main text \\ Epidemiology}

PV has an average age of onset of 40-60 years (Joly and Litrowski 2011). It has a prevalence of around 30,000 cases in the USA and an incidence of 1-10 new cases per 1 million people (Pemphigus. Pemphigus Pemphigoid Foundation (IPPF) 2014). It is a rare disease-especially in the pediatric population, but it needs no less study because it does affect patients and also does affect certain groups of people more than others. Ashkenazi Jews and people from India and the Middle East have higher rates of the disease (Pisanti et al. 1974). It is equally distributed among genders.

\section{Clinical presentation}

Patients can present with painful ulcerations of especially the buccal or palatine mucosa, but it can also present in the nose, genitals, anus, esophagus, and conjunctiva (Kavala et al. 2015; Kavala et al. 2011). In the skin, the bullae have a tendency to rupture, because the cellular interconnections are weakened by the autoimmune attack on desmogleins 1 and 3 (Stanley and Amagai 2006). Figure 1 shows PV of the oral mucosa as well as on the skin. The clinician can reproduce this rupturing or sloughing of the epidermis by putting 


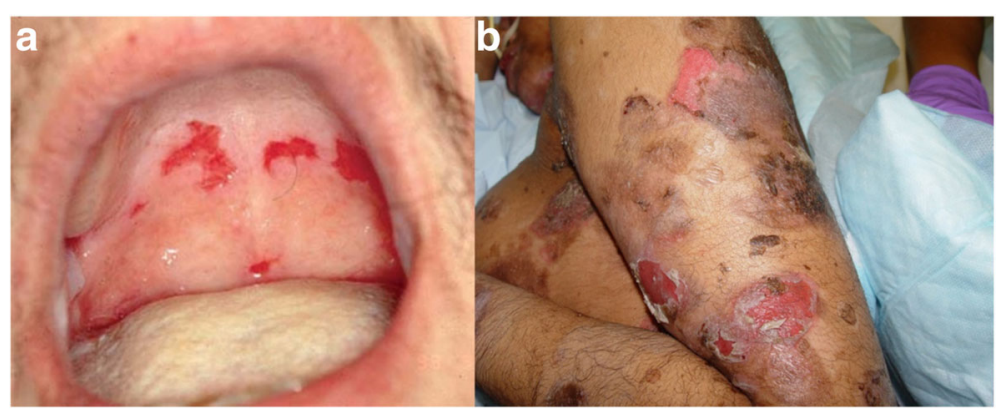

Fig. 1 PV on oral/skin mucosa. a: Pemphigus of the soft palate due to erosive disconnection of intraepidermal desmosomes. b: These are Pemphigus lesions of flaccid bullae that have ruptures on the upper arm. Loss of connections between keratinocytes due to autoantibodies against desmosome components render fluid buildup between these cells. They can rupture and slough away with time or manual pressure, such as seen in Nikolsky's sign

lateral pressure or traction on the bullae. When these slough off, it is referred to as a positive Nikolsky's sign (Venugopal and Murrell 2011). Because patients can have oral erosions, the pain can come with chewing and swallowing. This may lead to an avoidance of food and long-term nutritional deficiencies, which cause their own problems.

\section{Pathophysiology}

PV, as noted above, is an autoimmune disease. There are cell surface components known as desmoglein proteins which are components of the desmosomes in between keratinocytes. Keratinocytes are the cells which make up the layers of the epidermis. Particularly, in the stratum spinosum (called spinosum (Beutner and Jordon 1964) because the "spines"-which are desmosomes-can be seen between conjoining cells in this epidermal layer), desmosomes contribute to mechanical strength and integrity of and between cells, as well as cellular differentiation (Garrod and Chidgey 2008). The stratum spinosum also produces the keratin seen in the corny layer. This integrity of the cellular structure is part of the reason why skin is waterproof and tough. Antibodies to the two most common desmogleins-1 and 3-attack the epitope structure of these desmosomes and cause damage. Particularly, the immunoglobulin subclass of these autoantibodies is IgG4 (Ding et al. 1999; Bhol et al. 1995). Essentially, a type 2 hypersensitivity reaction takes place in which antibodies attach and destroy cell surface receptors. This leads to loss of integrity between keratinocytes in the stratum spinosum and loss of intercellular connectivity; this is referred to as acantholysis (Kumaran et al. 2013).

$\mathrm{PV}$, like other autoimmune diseases, is related to major histocompatibility complex (MHC) variation. $\mathrm{MHC}$ is a structure located on certain immune cells like macrophages and $\mathrm{B}$ cells which carry out immunologic functions, such as presenting to $\mathrm{T}$ cells and recognizing host and foreign antigens. Human leukocyte antigen (HLA) genes encode the MHC so that different variations in these genes cause a different immunologic function downstream (Janeway 1999). Particularly, PV is associated with HLA-DR4. This may explain why there is a positive association between type 1 diabetes mellitus, Hashimoto's thyroiditis, and rheumatoid arthritis (Parameswaran et al. 2015). However, HLA-DRB1 0402 is mostly associated with those of Ashkenazi Jewish descent (Kwon et al. 2001).

\section{Diagnosis}

There are other less common and less severe forms of pemphigus under the pemphigus umbrella, so weaving through the differential diagnosis involves a keen clinical acumen as well as the laboratory. To help confirm the diagnosis, other tests are run. After punch biopsy and histopathologic preparation, PV displays a particular cellular pattern. Because the problem is in between cells and not under the basal aspect of the cell, there is no space between the cell and the basement membrane. This leaves an intact, bottom layer of cells connected to the basement membrane. There is intraepidermal acantholysis seen (Bystryn and Rudolph 2005). If there was an autoimmune attack against hemidesmosomes on the basal side of the cell, there would be a loss of connection of the basal keratinocyte to the basement membrane, yielding a different and clinically less severe condition known as bullous pemphigoid. In the case of PV, the new pathologic space is in between the cells; this causes fluid to build up in places with lower intercellular integrity. Because the intercellular connections are lost-like a zipper, where the unzipped part is the damaged intercellular piece-and yet the cells are still intact on the basement membrane, they are said to have a tombstone pattern on histopathology (Baum et al. 2014). Figure 2 shows acantholysis and the "row of 


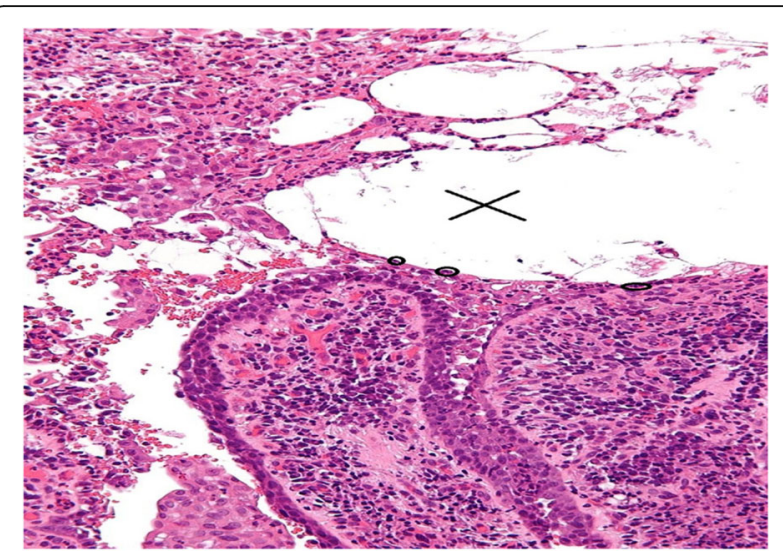

Fig. 2 PV histopathology. The "x" represents edema buildup between intraepidermal keratinocytes-also known as acantholysis- due to loss of adhesion between cells. Fluid will build up in these potential spaces when the chance arises. The bottom layer of cells (some have been circled) located underneath the edema are left intact and have been said to have a tombstone appearance. They represent the intact basal layer. Copyright (c) 2011 Michael Bonert (https:// commons.wikimedia.org/wiki/User:Nephron)

tombstones" pattern in PV. An increase in eosinophils can also be seen in the dermis.

Upon direct immunofluorescence of the skin or mucosal lesion, IgG can be seen as a "net-like" or reticular pattern where the IgG autoantibodies to desmogleins have been bound (Morrison 2001). This can be seen below in Fig. 3. An ELISA can be done to find autoantibodies to desmogleins 1 and 3 in the serum as well (Joly and Litrowski 2011). These can actually be tracked to assess remission and control of the disease (Abasq et al. 2009; Amagai et al. 1999).

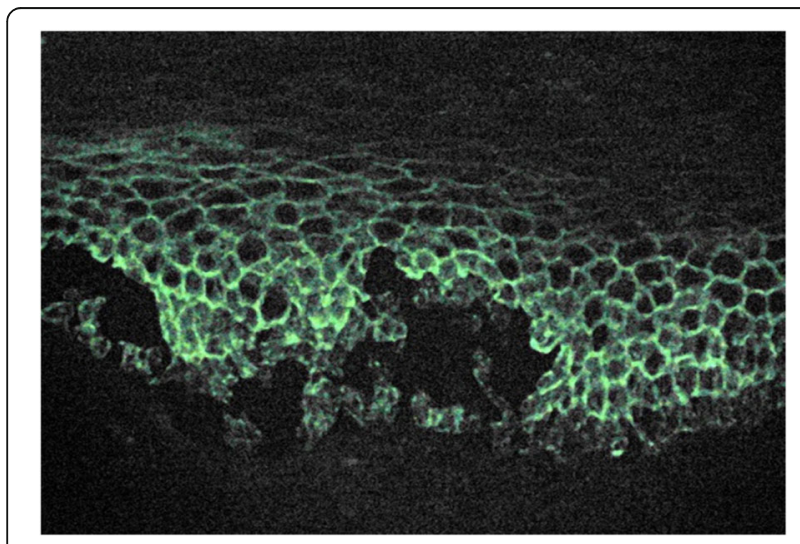

Fig. 3 PV direct immunofluorescence. IgG deposits intercellularly, along with intraepidermal acantholysis. The lgG deposition is represented by the hyperintense, brighter color distributed in a net-like or reticular pattern in between cells. The dark spaces nearby represent the fluid buildup that ensues as a result of loss of intercellular connection because of the lgG deposition. Courtesy of Wikipedia user: Emmanuelm

\section{Management}

Management of acute attacks and original management of the disease is with systemic glucocorticoids such as prednisolone, $1-1.5 \mathrm{mg} / \mathrm{kg}$ PO per day (Chams-Davatchi et al. 2007). The idea is to bring the patient into remission of their disease with steroids, using the smallest dosage possible; the reason for the smallest possible dosages is to avoid the possible comorbidities, such as Cushing's disease, osteoporosis, hypertension, and diabetes to name a few (Schacke 2002). As the patient begins to take systemic glucocorticoids, they can also be prescribed an adjunct treatment protocol with nonsteroidal but immunosuppressant drugs such as azathioprine and mycophenolate (Bystryn 1996). These are the same drugs used in many chemotherapy regimens for different cancers and other autoimmune conditions.

Being in remission is usually considered when the patient has been free of lesions for several weeks and with a negative Nikolsky's sign. At such a point, the systemic glucocorticoids are tapered down to a gradually decreasing dose. When the steroids can be stopped and the patient kept in remission, the other aforementioned adjunct drugs can begin to be tapered.

However, some of these drugs have potentially fatal side effects and do require monitoring. Azathioprine inhibits purine formation and can lead to myelosuppression. This requires quite frequent blood checkups and monitoring of kidney and liver function bi-weekly for the first 3 months and again periodically subsequently (Meggitt et al. 2011). Also because azathioprine is metabolized in the body by thiopurine methyltransferase (TPMT), the levels of this enzyme will need to be obtained upon initiating treatment (Jackson et al. 1997). Too little TPMT activity could result in gross overdosing of azathioprine and increased risk of adverse events such as myelosuppression. Mycophenolate has a safer side effect profile-aside from some gastrointestinal issues-than azathioprine, but it was found in randomized trials to have a lesser glucocorticoidsparing effect than azathioprine (Martin et al. 2011); this means that patients who use azathioprine as an adjunct to glucocorticoids needed to use less glucocorticoids due to azathioprine's superior glucocorticoid-sparing effect. Therefore, these drugs both have pros and cons, but each patient needs to be treated in a fashion that caters to his or her specific needs and pre-existing conditions.

Many patients go on to have recalcitrant PV after long periods of successful treatment with the aforementioned medications. A large majority do in fact achieve remission for at least 6 months if they have been treated with glucocorticoids and adjunct medications for close to 10 years; however, around $20-40 \%$ of patients do not ever go into remission and are considered refractory (Herbst and Bystryn 2000). PV can be a deadly disease, 
so enhanced treatment in these refractory patients is absolutely necessary. Rituximab has a place in treatment for PV based on the premise that a disease of antibodies is a disease of $B$ cells. Being an antibody against the $C D$ 20 antigen expressed on B cells. Rituximab can curb the disease, especially in refractory cases. Several case studies have shown rituximab to be efficacious (Hertl et al. 2008; Arin et al. 2005). Cyclophosphamide is also used in refractory cases, but it has an unsafe side effect profile (Saha et al. 2009). It can cause hemorrhagic cystitis. Because of its safety profile, it requires regular monitoring by the patient, which is an inconvenience. Plasmapheresis is also used occasionally by nonspecifically removing proteins from the blood, but it is costly and has its own unfavorable side effects, depending on the patient's condition. IVIG can also be used to dilute the autoantibodies with regular immunoglobulin.

The main reason that these interventions are used for recalcitrant disease instead of first line is simply their cost. Rituximab treatment can cost $\$ 16,000$ (Bomm et al. 2013). Patients may benefit by switching to a more practical, safer, and cheaper alternative. New advances in treatment of PV have emerged, specifically by way of innovative therapies such as subcutaneous veltuzumab injections. It has the same mechanism of action as rituximab, but it is cheaper and not as systemically absorbed. Some studies have shown that it can induce remission in patients after just two injections, 2 weeks apart (Ellebrecht et al. 2014). This drug may need more long-term controlled trials to further evaluate the efficacy for sustained remission, but it has been shown clinically to induce remission for patients who are refractory in their condition. With no monitoring and it being a cheaper, safer alternative, this could provide a new road for those suffering from recalcitrant disease.

\section{Conclusions}

$\mathrm{PV}$, while rare, is very distressing and painful for those who have it. The disease is not always isolated; many people also have concomitant chronic diseases to go along with it. This fact makes it urgent that PV be treated in a specific, patient-centered manner. We would not want someone who has diabetes mellitus and PV to take long-term glucocorticoids. The treatment plan should be tailored to the specific individual patient, following evidence-based recommendations mentioned herein. We can use the first-line drugs and a large majority of patients will be treated successfully; for the $20-40 \%$ of patients who are recalcitrant despite first-line and adjunctive agents, they must be treated with an innovative protocol. The use of new topical drugs and subcutaneous injections like veltuzumab has created a protocol whereby refractory PV patients can safely and effectively try a new, promising method of treatment. More numerous and long-term studies need to be done to evaluate the long-term benefits of these less systemic therapies. Perhaps one day, these drugs will be backed by enough evidence demonstrating their favorable safety profile and lower cost that they will be the first line of treatment instead of glucocorticoids. Until then, new randomized controlled trials will add power to an evergrowing body of evidence that there will always be newer, better therapies for those who suffer from PV.

\section{Abbreviations \\ IVIG: Intravenous immunoglobulin G; PV: Pemphigus vulgaris; \\ TPMT: Thiopurine methyltransferase}

\section{Acknowledgements}

Not applicable.

Funding

There was no funding for this review.

Availability of data and materials

Not applicable.

\section{Author's contributions}

WS worked on this review and did all of the researching groundwork as well as typing and formatting the work.

\section{Author's information}

William Sanders is a third year medical student at Georgia CampusPhiladelphia College of Osteopathic Medicine (PCOM). His interests include Dermatology and Internal Medicine.

Ethics approval and consent to participate

Not applicable.

\section{Consent for publication}

Not applicable.

\section{Competing interests}

The author declares that he has no competing interests.

\section{Publisher's Note}

Springer Nature remains neutral with regard to jurisdictional claims in published maps and institutional affiliations.

Received: 15 February 2017 Accepted: 20 September 2017

Published online: 03 December 2017

\footnotetext{
References

Abasq C, Mouquet H, Gilbert D, Tron F, Grassi V, Musette P, et al. ELISA Testing of Anti-Desmoglein 1 and 3 Antibodies in the Management of Pemphigus. Arch Dermatol. 2009;145(5):529-35.

Amagai M, Komai A, Hashimoto T, Shirakata Y, Hashimoto K, Yamada T, et al. Usefulness of enzyme-linked immunosorbent assay using recombinant desmogleins 1 and 3 for serodiagnosis of pemphigus. Br J Dermatol. 1999;140(2):351-7.

Arin M, Engert A, Krieg T, Hunzelmann N. Anti-CD20 monoclonal antibody (rituximab) in the treatment of pemphigus. Br J Dermatol. 2005;153(3):620-5.

Baum S, Sakka N, Artsi O, Trau H, Barzilai A. Diagnosis and classification of autoimmune blistering diseases. Autoimmun Rev. 2014;13(4-5):482-9.

Beutner E, Jordon R. Demonstration of Skin Antibodies in Sera of Pemphigus Vulgaris Patients by Indirect Immunofluorescent Staining. Exp Biol Med. 1964;117(2):505-10.

Bhol K, Natarajan K, Nagarwalla N, Mohimen A, Aoki V, Ahmed A. Correlation of peptide specificity and lgG subclass with pathogenic and nonpathogenic autoantibodies in pemphigus vulgaris: a model for autoimmunity. Proc Nat Acad Sci. 1995;92(11):5239-43.

Bomm L, Fracaroli T, Sodré J, Bressan A, Gripp A. Off-label use of rituximab in dermatology: pemphigus treatment. An Bras Dermatol. 2013;88(4):676-8.
} 
Bystryn J. The adjuvant therapy of pemphigus. Arch Derm. 1996;132(2):203-12. Bystryn J, Rudolph J. Pemphigus. Lancet. 2005;366(9479):61-73.

Chams-Davatchi C, Esmaili N, Daneshpazhooh M, Valikhani M, Balighi K, Hallaji Z, et al. Randomized controlled open-label trial of four treatment regimens for pemphigus vulgaris. J Am Acad Dermatol. 2007;57(4):622-8.

Ding X, Diaz L, Fairley J, Giudice G, Liu Z. The Anti-Desmoglein 1 Autoantibodies in Pemphigus Vulgaris Sera are Pathogenic. J Investig Dermatol. 1999;112(5):739-43.

Ellebrecht C, Choi E, Allman D, Tsai D, Wegener W, Goldenberg D, et al. Subcutaneous Veltuzumab, a Humanized Anti-CD20 Antibody, in the Treatment of Refractory Pemphigus Vulgaris. JAMA Dermatology. 2014;150(12):1331.

Garrod D, Chidgey M. Desmosome structure, composition and function. Biochim Biophys Acta Biomembr. 2008;1778(3):572-87.

Herbst A, Bystryn J. Patterns of remission in pemphigus vulgaris. J Am Acad Dermatol. 2000;42(3):422-7.

Hertl M, Zillikens D, Borradori L, Bruckner-Tuderman L, Burckhard H, Eming R, et al. Recommendations for the use of rituximab (anti-CD20 antibody) in the treatment of autoimmune bullous skin diseases. J Dtsch Dermatol Ges. 2008;6(5):366-73

Jackson A, Hall A, McLelland J. Thiopurine methyltransferase levels should be measured before commening patients on azathioprine. Br J Dermatol. 1997;136(1):133-4.

Janeway C. Immunobiology: The Immune System in Heath and Disease. 5th ed. London: Harcourt Brace and Company; 1999.

Joly P, Litrowski N. Pemphigus group (vulgaris, vegetans, foliaceus, herpetiformis, brasiliensis). Clin Dermatol. 2011;29(4):432-6.

Kavala M, Altıntaş S, Kocatürk E, Zindancı I, Can B, Ruhi C, et al. Ear, nose and throat involvement in patients with pemphigus vulgaris: correlation with severity, phenotype and disease activity. J Eur Acad Dermatol Venereol. 2011;25(11):1324-7.

Kavala M, Topaloğlu Demir F, Zindanci I, Can B, Turkoğlu Z, Zemheri E, et al. Genital involvement in pemphigus vulgaris (PV): Correlation with clinical and cervicovaginal Pap smear findings. J Am Acad Dermatol. 2015;73(4):655-9.

Kumaran M, Kanwar A, Seshadri D. Acantholysis revisited: Back to basics. Indian J Dermatol Venereol Leprol. 2013;79(1):120.

Kwon O, Brautbar C, Weintrob N, Sprecher E, Saphirman C, Bloch K, et al. Immunogenetics of HLA class II in Israeli Ashkenazi Jewish, Israeli nonAshkenazi Jewish, and in Israeli Arab IDDM patients. Hum Immunol. 2001;62(1):85-91.

Martin L, Werth V, Villaneuva E, Murrell D. A systematic review of randomized controlled trials for pemphigus vulgaris and pemphigus foliaceus. J Am Acad Dermatol. 2011;64(5):903-8.

Meggitt S, Anstey A, Mohd Mustapa M, Reynolds N, Wakelin S. British Association of Dermatologists' guidelines for the safe and effective prescribing of azathioprine 2011. Br J Dermatol. 2011;165(4):711-34.

Morrison L. Direct immunofluorescence microscopy in the diagnosis of autoimmune bullous dermatoses. Clin Dermatol. 2001;19(5):607-13.

Mustafa M, Porter S, Smoller B, Sitaru C. Oral mucosal manifestations of autoimmune skin diseases. Autoimmun Rev. 2015;14(10):930-51.

Parameswaran A, Attwood K, Sato R, Seiffert-Sinha K, Sinha A. Identification of a new disease cluster of pemphigus vulgaris with autoimmune thyroid disease, rheumatoid arthritis and type I diabetes. Br J Dermatol. 2015;172(3):729-38.

Pemphigus. Pemphigus Pemphigoid Foundation (IPPF) 2014. http://www.pemphigus. org/research/clinically-speaking/pemphigus/. Accessed 11 Feb 2017.

Pisanti S, Sharav Y, Kaufman E, Posner L. Pemphigus vulgaris: Incidence in Jews of different ethnic groups, according to age, sex, and initial lesion. Oral Surg Oral Med Oral Pathol. 1974;38(3):382-7.

Razzaque Ahmed A, Moy R. Death in pemphigus.J Am Acad Dermatol. 1982:7(2):221-8.

Saha M, Powell A, Bhogal B, Black M, Groves R. Pulsed intravenous cyclophosphamide and methylprednisolone therapy in refractory pemphigus. Br J Dermatol. 2009;162(4):790-7.

Schacke H. Mechanisms involved in the side effects of glucocorticoids. Pharmacol Ther. 2002;96(1):23-43.

Stanley J, Amagai M. Pemphigus, Bullous Impetigo, and the Staphylococcal Scalded-Skin Syndrome. N Engl J Med. 2006;355(17):1800-10,

Venugopal S, Murrell D. Diagnosis and Clinical Features of Pemphigus Vulgaris. Dermatol Clin. 2011;29(3):373-80.

\section{Submit your next manuscript to BioMed Central and we will help you at every step:}

- We accept pre-submission inquiries

- Our selector tool helps you to find the most relevant journal

- We provide round the clock customer support

- Convenient online submission

- Thorough peer review

- Inclusion in PubMed and all major indexing services

- Maximum visibility for your research

Submit your manuscript at www.biomedcentral.com/submit
Biomed Central 